\title{
Mineração
}

\section{Adsorção de coletores aniônicos em silicatos}

\author{
Paulo Roberto de Magalhães Viana \\ Professor Substituto, Departamento de Engenharia de Minas da EEUFMG \\ E-mail:pviana@demin.ufmg.br \\ Armando Corrêa de Araujo \\ Professor Adjunto, Departamento de Engenharia de Minas da EEUFMG \\ E-mail:armando@demin.ufmg.br \\ Antônio Eduardo Clark Peres \\ Professor Adjunto, Departamento de Engenharia Metalúrgica da EEUFMG \\ E-mail: aecperes@demet.ufmg.br
}

\section{Resumo}

A compreensão dos mecanismos de adsorção de coletores é de fundamental importância para a otimização e eventualmente a viabilização do processo de flotação. Embora os coletores aniônicos estejam entre os principais reagentes usados em diversos sistemas de flotação, poucos estudos de adsorção desses reagentes em silicatos têm sido realizados, quando se comparam tais estudos com o grande número de estudos desenvolvidos com minerais levemente solúveis e óxidos. O presente trabalho faz uma revisão concisa dos mecanismos de adsorção de coletores aniônicos em silicatos, incluindo ácidos carboxílicos, sulfatos e sulfonatos. Destacam-se os ácidos carboxílicos, que, devido à facilidade com que formam sais insolúveis com diversos cátions metálicos, têm tido amplo emprego na flotação de silicatos.

Palavras-chave: Flotação, silicatos, adsorção de coletores.

\begin{abstract}
The understanding of the adsorption mechanisms of collectors is of primary importance for the optimization and, eventually, the feasibility of the flotation process. Although anionic collectors are included in the main reagents used in various flotation systems, only a few studies of adsorption of these reagents in silicate flotation systems have been carried out when compared with the large number of studies developed with sparingly soluble minerals and oxides. The present work makes a concise revision of the adsorption mechanisms for the main anionic silicate collectors, carboxylic and sulfonic acids and sulfates. Carboxylic acids are emphasized due to their ability to form insoluble salts with metallic cations.
\end{abstract}

Keywords: Flotation, silicate, collector adsorption. 


\section{Introdução}

Aminas e ácidos carboxílicos são usados em diversos sistemas de flotação e são os principais reagentes usados na flotação de silicatos. Com a exceção dos sulfetos e elementos nativos, as aminas ou os ácidos carboxílicos estão presentes na flotação de inúmeros minerais de todos os demais grupos mineralógicos. O estudo da adsorção desses reagentes é de fundamental importância para o processo de flotação, pois a compreensão da natureza e a forma da adsorção podem gerar consequiências práticas na indústria como melhoria de seletividade, índices de recuperação e eventualmente redefinição de rotas de processo. Os modelos de adsorção de reagentes são estruturados normalmente nas interações entre sítios superficiais, os próprios reagentes e outras espécies iônicas ou moleculares presentes no sistema que se estuda. Uma divisão dos modelos existentes, que se aplicam aos silicatos, é proposta na Tabela 1.

\section{Adsorção física de coletores aniônicos em silicatos}

Os silicatos apresentam nenhuma ou pouca solubilidade em faixa de $\mathrm{pH}$ ácida, não respondendo à flotação com coletores aniônicos em faixa de $\mathrm{pH}$, ácida ou alcalina, acima do PZC. A adsorção física de coletores aniônicos em silicatos ocorre em valor de $\mathrm{pH}<\mathrm{pH}_{\mathrm{PZC}}$. Cases (1967) mostra a flotação com coletores aniônicos (dodecilsulfato e sulfo-

Tabela 1 - Adsorção de coletores em silicatos, Viana (2004).

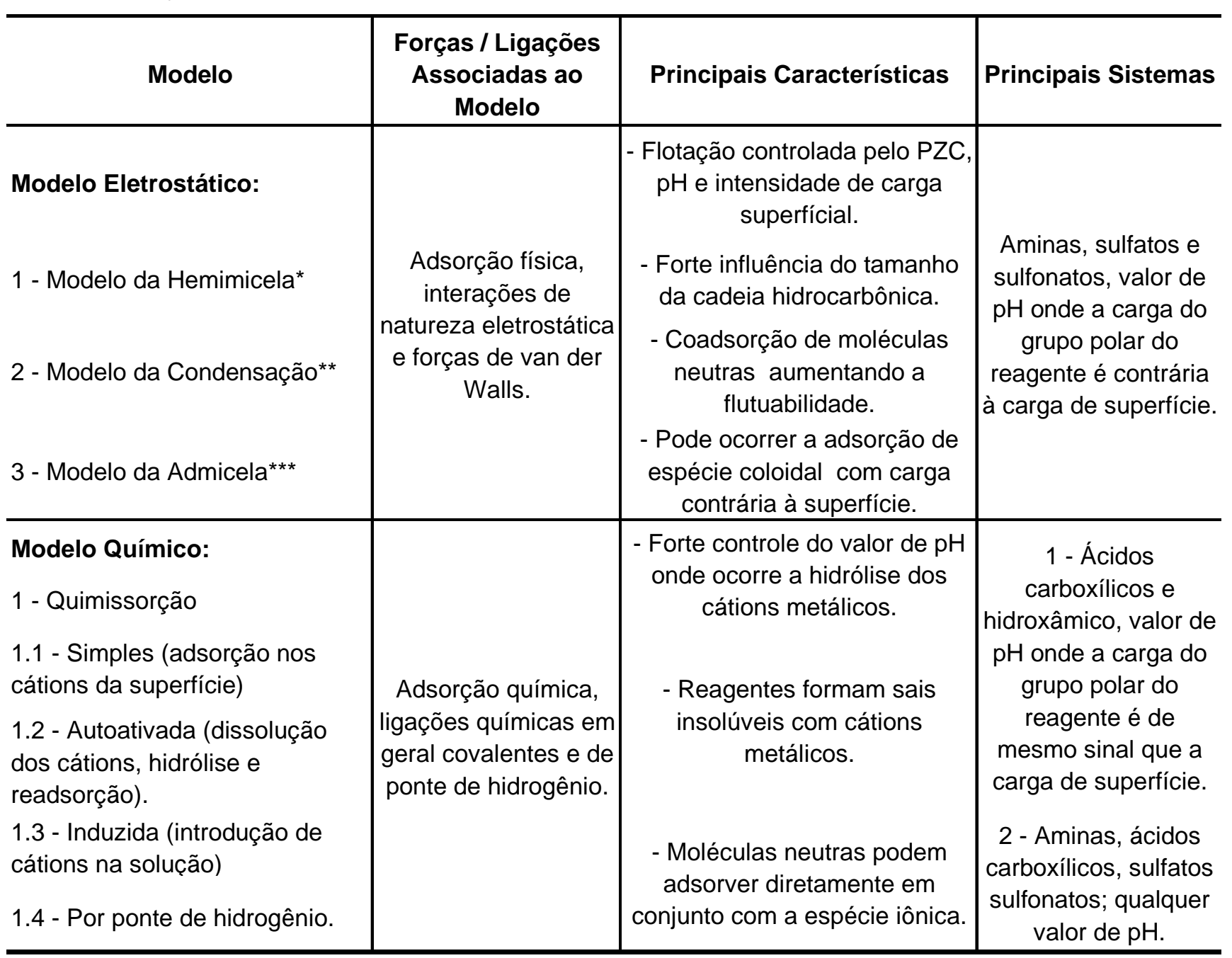

*Modelo da Hemimicela (HM): adsorção em uma camada, interações eletrostáticas e ligações laterais.

**Modelo da Condensação Bidimensional (CB): interações normais, laterais e termos entrópicos para a camada adsorvida. Agregação controlada por heterogeneidade da superfície e camadas bidimensionais.

*** Modelo da Admicela(AD): surfatante/íons contrários agregam-se em camadas duplas incompletas. 
Paulo Roberto de Magalhães Viana et al.

nato) em valores de $\mathrm{pH}<\mathrm{pH}_{\mathrm{PZC}}$. Os minerais são a granada, zirconita, distênio e berílio com $\mathrm{pH}_{\mathrm{PZC}}$ de 5,8, 4,4, 6,2 e 3,0, respectivamente. A flotação é incipiente em valor de $\mathrm{pH}>\mathrm{pH}_{\mathrm{PZC}}$ com os coletores aniônicos e efetiva com coletor catiônico, indicando adsorção promovida por interações eletrostáticas, Figura 1.

A dissociação de sulfatos e sulfonatos estende-se a valores extremamente baixos de $\mathrm{pH}$, enquanto que o $\mathrm{pKa}$ dos ácidos carboxílicos está na faixa de 4 a 5. Laskowski (1987) constatou a formação de um precipitado coloidal dos ácidos carboxílicos em torno de $\mathrm{pH} 3$ a 4 (ver Figura 2), que, provavelmente, impede a adsorção em $\mathrm{pH}$ muito ácido, uma vez que esse precipitado tem carga positiva nessa faixa de $\mathrm{pH}$, onde vários silicatos e óxidos já apresentam carga de superfície também positiva.

A formação do precipitado coloidal poderia explicar o aumento da flutuabilidade no sistema espodumênio/oleato em torno de $\mathrm{pH} 4$, pois o PZC do espodumênio ocorre em $\mathrm{pH}$ 2,5, possibilitando, desta maneira, a adsorção física do oleato sem ativação por cátions metálicos (Figura 4). Silicatos e óxidos como a sillimanita, zirconita, cromita (Figura 3) e pirolusita também mostram um aumento na flutuabilidade com oleato em torno de $\mathrm{pH}$ 4. Esse aumento tem sido justificado pela formação de hidroxicomplexo de alumínio(pirolusita não tem alumínio), hidroxicomplexo de ferro ou simplesmente adsorção física. Parece ser mais adequado atribuir tal fato simplesmente à adsorção física, posto que os minerais citados têm PZC entre pH 5,5 a 7,5.

\section{Adsorção química de coletores aniônicos em silicatos}

A adsorção de coletores aniônicos em silicatos, ocorrendo em valores de $\mathrm{pH}$ maiores que o $\mathrm{pH}_{\mathrm{PZC}}$, é explicada pela quimissorção de espécies iônicas ou moleculares do coletor. Fuerstenau e Han (2002) indicam que a quimissorção pode ocorrer pela adsorção direta do coletor em sítios de cátions metálicos na superfície do mineral ou ser promovida pela auto-ativação, onde cátions metálicos deixam a superfície, são hidrolizados e readsorvem ou, ainda, por cátions introduzidos forçadamente no sistema. Em um ácido carboxílico, o carbono do grupo carbonila tem deficiência em elétrons e está ligado a um segundo oxigênio conectado ao átomo de hidrogênio. $\mathrm{O}$ arranjo dessa configuração é tal que a densidade eletrônica é deslocada do hidrogênio do grupo hidroxila em direção ao grupo carbonila, levando à perda de um próton e conseqüente ionização estabilizada por ressonância. Essa estabilização por ressonância gera o caráter de ácido fraco dos ácidos carboxílicos e possibilita a formação de sais insolúveis, quando reagem com cátions como $\mathrm{Fe}^{2+}$,

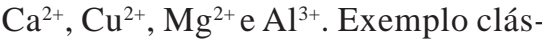
sico de ativação por cátions é a flotação de quartzo com coletor aniônico, que é incipiente ou nula em valor de $\mathrm{pH}>\mathrm{pH}_{\mathrm{PZC}}(\sim 2)$. A Figura 4 mostra os limites da flotação do quartzo com sulfo-

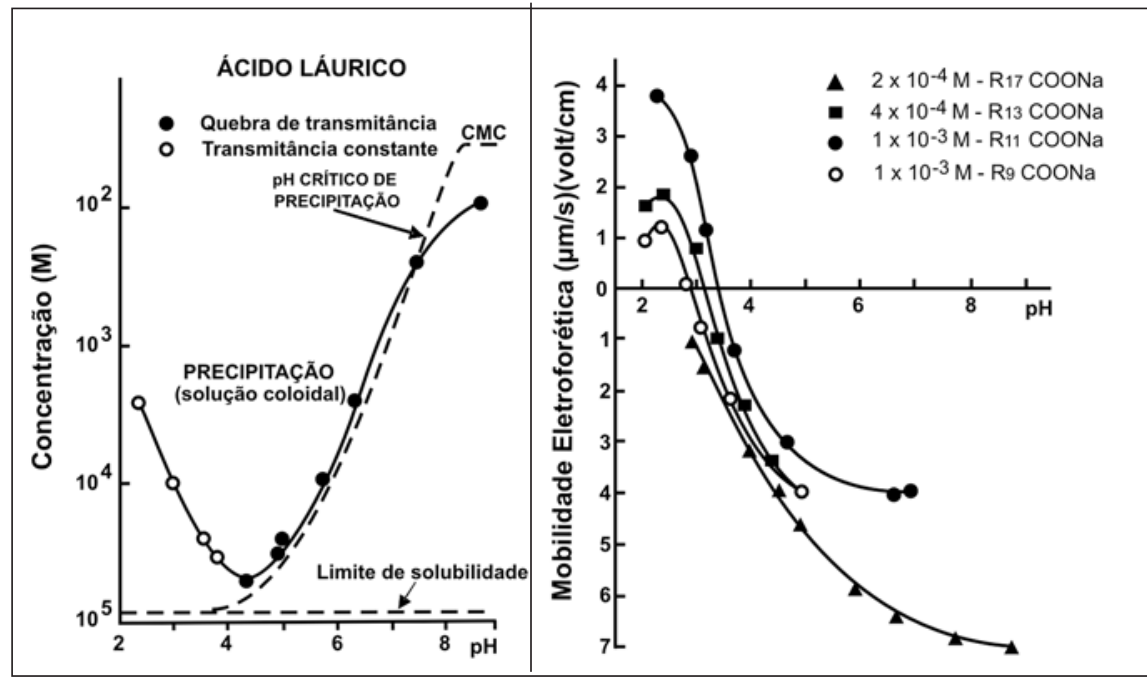

Figura 2 - Diagrama de equilíbrio/mobilidade eletroforética (Laskowski, 1987).
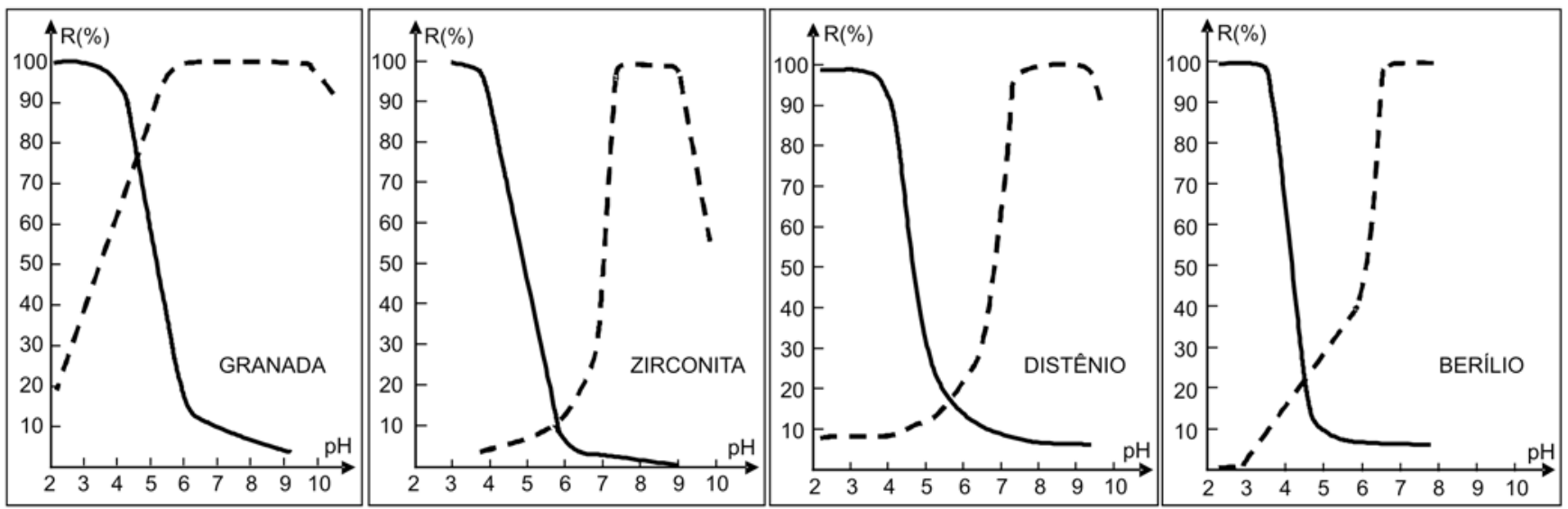

Figura 1 - Recuperação de granada, zirconita, distênio e berilo em função do $\mathrm{pH}\left(-\mathrm{R}_{12} \mathrm{OSO}_{2}{ }^{-} \mathrm{Na}^{+}\right.$e --- $\left.\mathrm{R}_{12} \mathrm{NH}_{3}{ }^{+} \mathrm{Cl}^{-}\right)(\mathrm{Cases}, 1967)$. 
nato, ativada por cátions, na faixa de $\mathrm{pH}$ onde ocorre a hidrólise dos mesmos.

Palmer (1975) mostrou a ativação de óxidos e silicatos em estudo em sistemas cromita/ácido oléico e crisocola-rodonita/hidroxamato. O mecanismo sugerido é a dissolução do cátion $\mathrm{Mn}^{+}$, hidrólise em solução, adsorção do hidroxicomplexo via ligações de hidrogênio com os grupos $\mathrm{OH}^{-}$da superfície ou desprotonação do hidroxicomplexo pelos grupos $\mathrm{OH}$ da superfície, formação de água, adsorção do hidroxamato no sítio do cátion metálico e posterior adsorção de um precipitado metal-surfatante via interações hidrofóbicas. A Figura 5 mostra a reversão da carga da rodonita na presença de íons $\mathrm{Mn}^{2+}$ e a resposta à flotação da rodonita nessa situação.

Vijaya (2002) mostra a necessidade de hidrólise do íon metálico $\mathrm{Al}^{3+}$ na adsorção de oleato na superfície de sillimanita. O máximo de adsorção do oleato ocorreu em pH 7,5 e a reação de quimissorção proposta é representada pela equação 1 .

$\mathrm{AlOH}_{\text {superfície }}+\mathrm{RCOOH} \leftrightarrow \mathrm{AlOOCR}_{\text {superfície }}+\mathrm{H}_{2} \mathrm{O}$

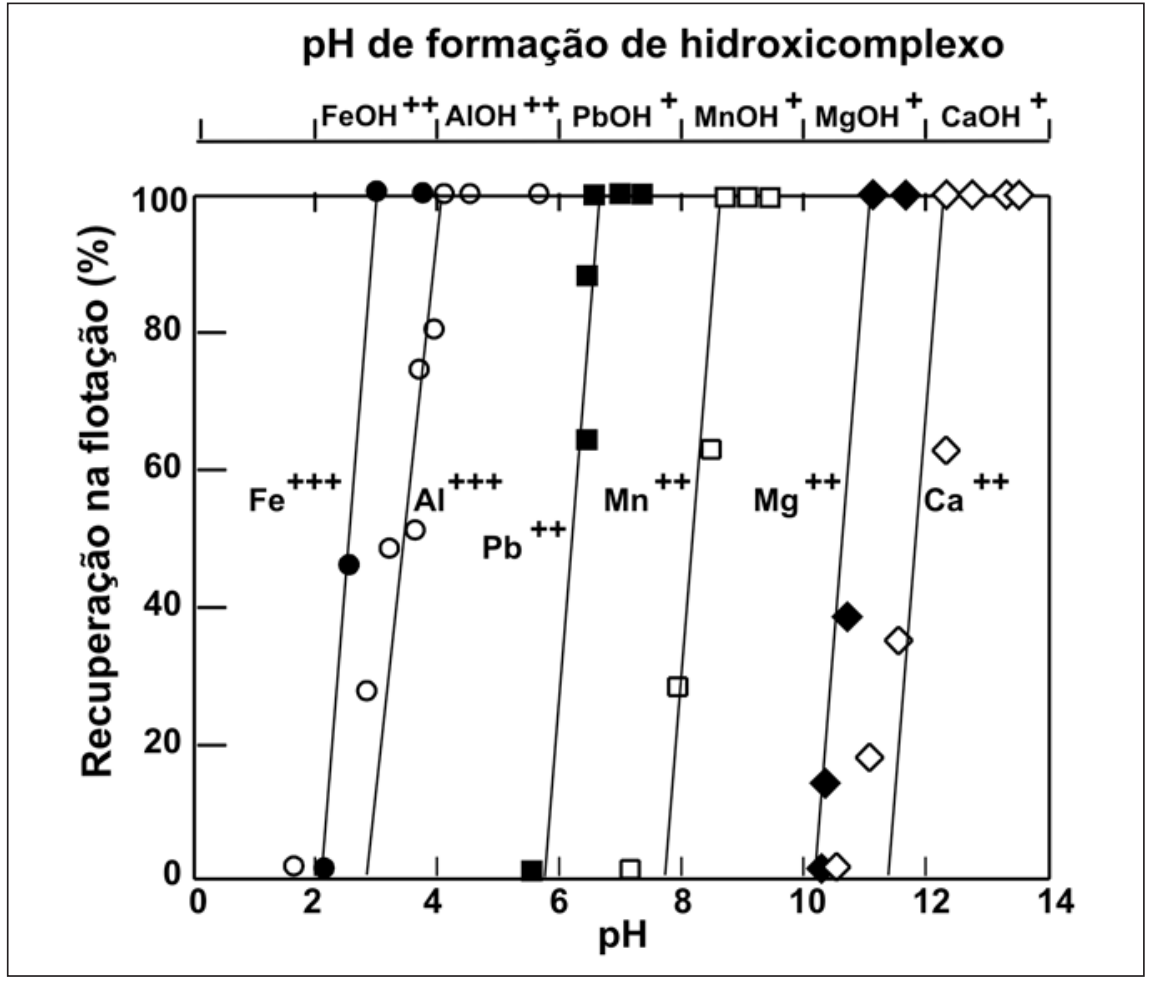

Considerou-se que os prótons do ácido oléico se polarizavam em direção aos grupos hidroxila da superfície com a formação de moléculas de água. Dessa maneira, os grupos superficiais $\mathrm{OH}$ facilitavam a desprotonação do ácido, promovendo sua adsorção. Em um experimento isolado, o ácido oléico foi adicionado em uma solução com íons $\mathrm{Al}^{3+} \mathrm{e}$, mesmo após várias horas, para se alcançar o equilíbrio da reação, não foi detectado oleato de alumínio precipitado. Como a diminuição do ácido oléico foi observada em valor de $\mathrm{pH}$, onde o hidróxido de alumínio é formado, inferiuse que o ácido oléico só reage na presença do hidróxido do metal. Kwang (2003) indica, no sistema espodumênio/ oleato, um pico primário na adsorção em pH 8, Figura 6. A quimissorção nos sítios superficiais de alumínio foi considerada como sendo responsável pelo máximo de flutuabilidade em pH 8. A adição de íons alumínio na solução em pH 8 não

Figura 4 - Ativação por cátions metálicos (Fuerstenau, 2002).

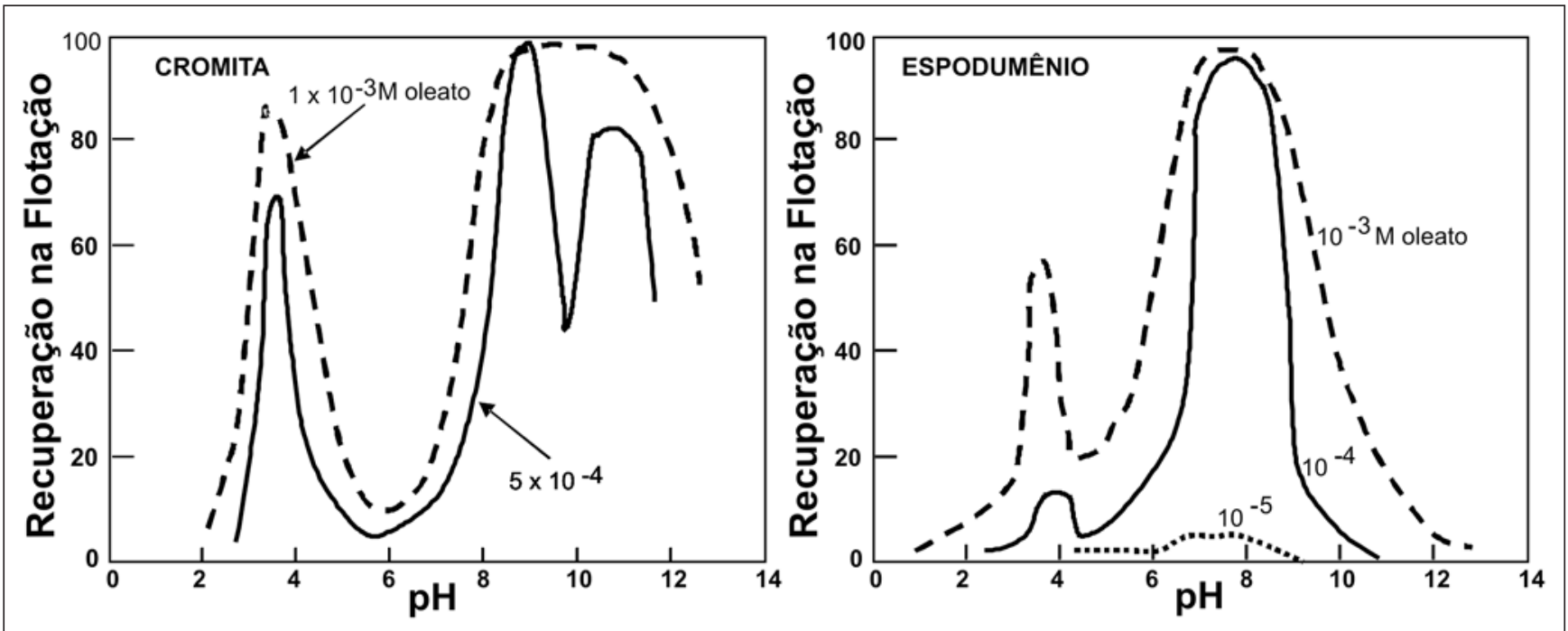

Figura 3 - Flotação de cromita e espodumênio com oleato (Palmer, 1975; Kwang, 2003). 
Paulo Roberto de Magalhães Viana et al.

modificou a flotação, mas a lixiviação prévia do espodumênio em pH 12 ampliou a faixa de flotação em torno de $\mathrm{pH}$ 8. Foi sugerido que a lixiviação promoveu a quebra da estrutura cristalina, levando a um aumento da densidade de sítios de alumínio na superfície. A seletividade na flotação do espodumênio, em relação a outros silicatos, como muscovita e feldspatos em $\mathrm{pH} 8$, foi atribuída à adsorção preferencial do oleato nos sítios de alumínio mais expostos na estrutura cristalina do espodumênio do que em relação aos sítios de alumínio na estrutura cristalina dos minerais citados.

Em estudo sobre a adsorção de ácido oléico na superfície de fenacita, ativada por HF, e na superfície de berilo, Peck(1967) mostra a quimissorção em ambos os casos. Para a fenacita, a quimissorção ocorreria através da formação de um sal do ácido oléico, no qual o cátion é o berílio, conforme ilustrado pela reação da equação 2.

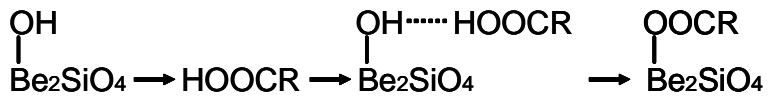

Para o berilo, o mecanismo proposto é a troca de grupo $\mathrm{OH}^{-}$da superfície pelo íon $\mathrm{F}^{-}$e a formação de ponte de hidrogênio, entre o íon $\mathrm{F}^{-}$e o hidrogênio do grupo carboxila, equação 3 .

$\mathrm{F}$

$\overbrace{\mathrm{Be}_{3} \mathrm{Al}_{2} \mathrm{Si}_{6} \mathrm{O}}{ }_{18} \quad \mathrm{HOOCR} \rightarrow \overbrace{\mathrm{Be}_{3} \mathrm{Al}_{2} \mathrm{Si}_{6} \mathrm{O}_{18}}$

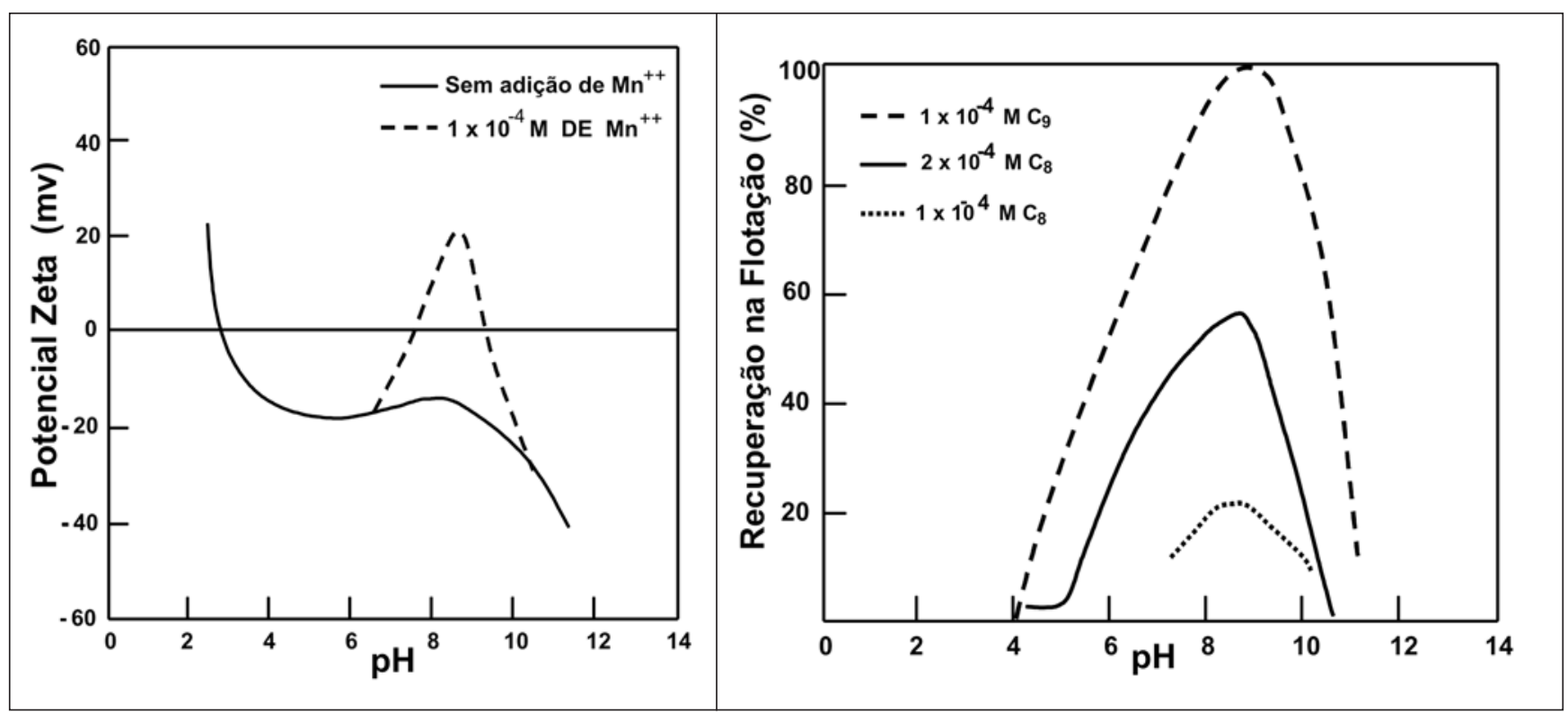

Figura 5 - $\mathrm{MnSiO}_{3}$, potencial zeta/flotabilidade (Palmer, 1975).

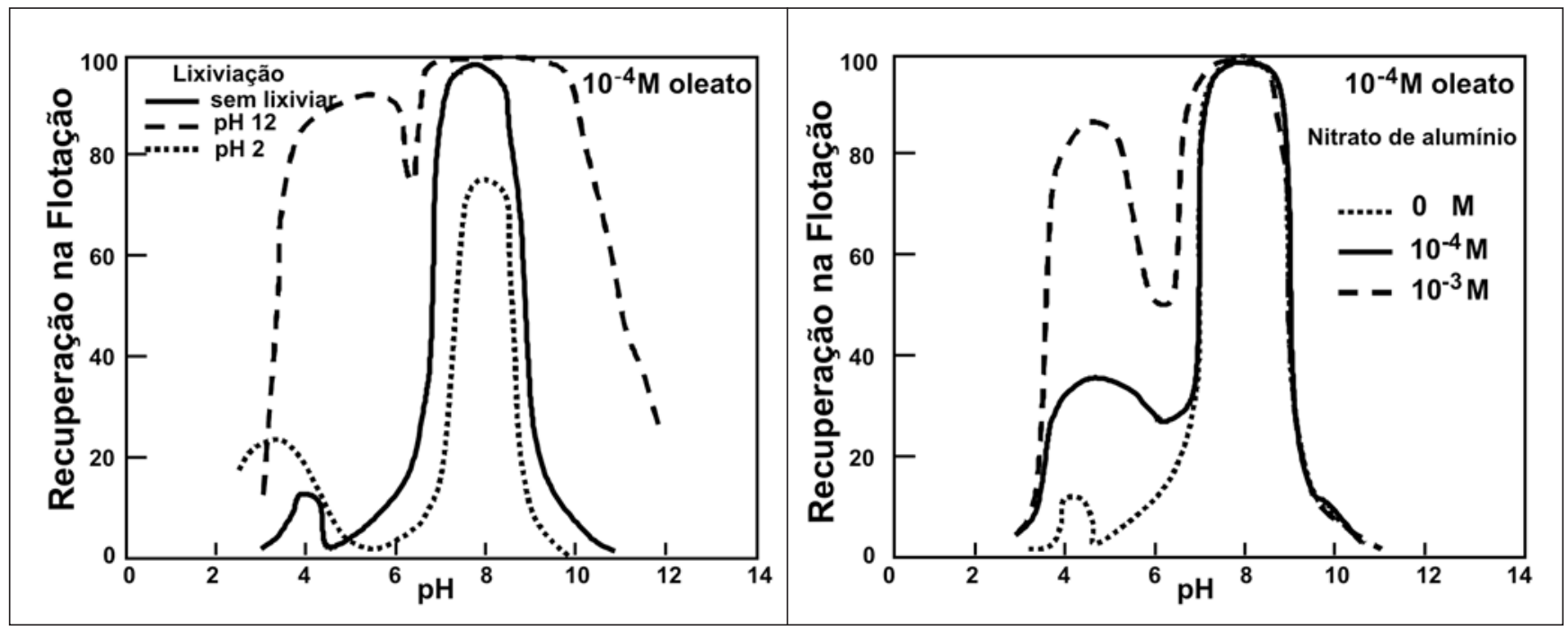

Figura 6 - Flotação de espodumênio após lixiviação em pH 12 e na presença de nitrato de alumínio (Kwang, 2003). 
Adsorção de coletores aniônicos em silicatos

Tabela 2 - Mecanismo de adsorção de silicatos (Viana, 2004).

\begin{tabular}{|c|c|c|c|c|c|c|c|c|}
\hline \multirow{2}{*}{ Mineral } & \multirow{2}{*}{ PZC } & \multirow{2}{*}{ Reagente } & \multirow{2}{*}{$\mathrm{pH}$} & \multirow{2}{*}{ Ativação } & \multirow{2}{*}{$\begin{array}{c}\text { Mecanismo } \\
\text { Adsorção }\end{array}$} & \multicolumn{3}{|c|}{ Metodologia } \\
\hline & & & & & & P.Z. & M. F. & I.V. \\
\hline Quartzo & 1,4 a 2,3 & Ácido carboxílico & 11 & $\mathrm{Ca}^{2+}$ & Quimissorção & & Sim & \\
\hline Microclina & 1,7 a 2,4 & Oleato $\mathrm{Na}$ & 2 a 6 & & Não flutua & Sim & Sim & Sim \\
\hline Albita & 1,9 a 2,3 & Oleato $\mathrm{Na}$ & 2 a 6 & & Não flutua & Sim & Sim & Sim \\
\hline Muscovita & 1,0 a 3,2 & Oleato $\mathrm{Na}$ & 8 & & Quimissorção & Sim & Sim & \\
\hline Lepidolita & 1,6 a 2,6 & Oleato $\mathrm{Na}$ & 9 & & Quimissorção & Sim & Sim & \\
\hline Rodonita & 2,8 & Hidroxamato $\mathrm{K}$ & 8,5 & $\mathrm{MnOH}^{+}$ & Quimissorção & Sim & Sim & Sim \\
\hline Crisocola & 2 & Hidroxamato $\mathrm{K}$ & 6 & $\mathrm{CuOH}^{+}$ & Quimissorção & Sim & Sim & Sim \\
\hline Espodumênio & 2,6 & Oleato $\mathrm{Na}$ & $4^{*}$ e 8 & & $\begin{array}{l}{ }^{*} \text { Fisissorção } \\
\text { Quimissorção }\end{array}$ & Sim & Sim & Sim \\
\hline Augita & 2,7 a 4,5 & Oleato & 3,8 e 11 & $\begin{array}{l}\mathrm{FeOH}^{+} \\
\mathrm{MgOH}^{+}\end{array}$ & Quimissorção & & Sim & \\
\hline Fenacita & Nesossil. & Ácido oléico & 6,3 & & Quimissorção & & Sim & Sim \\
\hline Berilo & 2,7 a 3,4 & Sulfonato $\mathrm{Na}$ & $<4$ & & Fisissorção & Sim & Sim & \\
\hline Berilo & 2,7 a 3,4 & Ácido oléico & $>4$ & & Não flutua & & Sim & \\
\hline Berilo & 2,7 a 3,4 & Ácido oléico & 7 & $\mathrm{~F}^{-}$ & Quimissorção & & Sim & Sim \\
\hline Turmalina & 3,9 & $\mathrm{Di} /$ tricarboxilato $\mathrm{Na}$ & 5 a 10 & & Quimissorção & & Sim & Sim \\
\hline Granada & 4,4 & Sulfonato $\mathrm{Na}$ & $<4$ & & Fisissorção & Sim & Sim & \\
\hline Zirconita & 5,8 & Sulfonato $\mathrm{Na}$ & $<3,5 / 4^{*}$ & & $\begin{array}{l}\text { *Fisissorção } \\
\text { Fisissorção }\end{array}$ & Sim & Sim & \\
\hline Distênio & 6,2 a 7,9 & Sulfonato $\mathrm{Na}$ & $<4$ & & Fisissorção & Sim & Sim & \\
\hline Sillimanita & 5,6 a 8,0 & Ácido oléico & $4^{*}$ e 7,5 & & $\begin{array}{l}\text { *Fisissorção } \\
\text { Quimissorção }\end{array}$ & Sim & & Sim \\
\hline
\end{tabular}

\section{Comentários Finais}

Um resumo do principal mecanismo de adsorção com coletores aniônicos, para 16 silicatos, é apresentado na Tabela 2. Algumas observações sobre a tabela são destacadas a seguir.

- Quando o principal mecanismo de adsorção é a quimissorção, com exceção da magnesita, ele sempre ocorre em faixa de $\mathrm{pH}$ acima do $\mathrm{pH}$ do $\mathrm{PZC}$.

- A adsorção física ocorre, na grande maioria das vezes, em valores de $\mathrm{pH}$ abaixo do $\mathrm{pH}$ do PZC.

- Os recursos utilizados para os estudos de adsorção foram microflotação (M.F.), medidas de potencial zeta (P.Z.) e espectroscopia infravermelha (I.V).

\section{Referências Bibliográficas}

CASES J. M. Les phénomènes physico-chimiques à l'interface application au procédé de la flottation. France: Nancy, Faculté des Sciences de L’Université de Nancy, 1967. 119p. (Grade de Docteur Ès-Sciences Phisiques).

CASES, J. M., VILLIERAS, F. Thermodynamic model of ionic and nonionic surfactant adsorption-abstraction on heterogeneous surfaces. Langmuir, v. 8, p. 1251-1264, 1992.

FUERSTENAU, D. W. Correlation of contact angles, adsorption density, zeta potencial and flotation rate. Transactions AIME, v. 208, p. 1365-1367, 1957.

FUERSTENAU, M. C., HAN, K. N. Metal-Surfactant precipitation and adsorption in froth flotation. Journal of Colloid and Interface Science, v. 256, p. 175-182, 2002.

HARWELL, J. H., HOSKINS, J. C., SCHECHTER, R. S., WADE W. H. Pseudophase separation model for surfactant adsorption: isomerically pure surfactant. Langmuir, p. 251-262, 1985.

KWANG, S. M., FUERSTENAU, D. W. Surface crystal chemistry in selective flotation of spodumene $\left(\mathrm{LiAl}\left[\mathrm{SiO}_{3}\right]_{2}\right)$ from other aluminosilicates. International Journal of Mineral Processing, v. 72, p. 11-24, 2003.

LASKOWSKI J. S., VURDELA R. M. Positively charged colloidal especies in aqueous anionic surfactant solutions. Colloids and Surfaces. Amsterdam. v. 22, p77-80, 1987.

PALMER, B. R., GUTIERREZ, B., FUERSTENAU, M. C., APLAN, F. F. Mechanisms involved in the flotation of oxides and silicates with anionic collectors: Parts 1 and 2. Transactions AIME, v. 258, p. 257-263, 1975.

PECK, A. S., WADSWORTH, M. E. An infrared study of the flotation of phenacite with oleic acid. Transactions AIME, v. 238, p. 245-248, 1967.

PECK, A. S., WADSWORTH, M. E. An infrared study of the activation and flotation of beryl with hydrofluoric and oleic acid. Transactions AIME, v. 238, p. 264-268, 1967.

VIANA, P.R.M. Flotação de silicato de lítio, feldspato e quartzo com coletores aniônicos, cationnicos, anfotéricos e mistura de coletores. Belo Horizonte: Universidade Federal de Minas Gerais, Curso de PósGraduação em Engenharia Metalúrgica e de Minas, 2004. (Proposta de Tese de Doutorado).

VIJAYA, K. T. V., S. PRABHAKAR BHASKAR, R. G. Adsorption of oleic acid at sillimanite/water interface. Journal of Colloid and Interface Science, v.247, p. 275-281, 2002.

Artigo recebido em 22/11/2004 e aprovado em $06 / 07 / 2005$. 